\title{
Numerical simulation of grain size distribution in two-phase polycrystalline materials
}

\author{
Rifa J. El-Khozondar ${ }^{1, ~ *, ~ D a n a ~ Z o ̈ l l n e r ², ~ K l a u s ~ K a s s n e r ~}{ }^{3}$ \\ ${ }^{1}$ Physics Department, Al-Aqsa University, Gaza, Palestine \\ ${ }^{2}$ Institute for Experimental Physics, Otto von Guericke University Magdeburg, Magdeburg, Germany \\ ${ }^{3}$ Institute for Theoretical Physics, Otto von Guericke University Magdeburg, Magdeburg, Germany \\ Email address: \\ rifa20012002@yahoo.com (R. J. El-Khozondar), dana.zoellner@ovgu.de (D. Zöllner), Klaus.Kassner@ovgu.de (K. Kassner)
}

\section{To cite this article:}

Rifa J. El-Khozondar, Dana Zöllner, Klaus Kassner. Numerical Simulation of Grain Size Distribution in Two-Phase Polycrystalline Materials. International Journal of Materials Science and Applications. Vol. 3, No. 6, 2014, pp. 381-390. doi: 10.11648/j.ijmsa.20140306.26

\begin{abstract}
Numerical simulations based on the Monte Carlo Potts model are used to study the temporal change of the grain size distribution of two-phase polycrystalline materials, where both phases grow simultaneously. After a sufficiently long time, grain growth in such two-phase systems can be characterized by a self-similar scaled grain size distribution function and an associated growth law. In particular, the grain size distribution is analyzed for a broad range of second phase volume fractions and found to vary with the volume fraction such that the size distribution becomes narrower and higher peaked with decreasing volume fraction of the second phase, where particularly the normal distribution function describes the simulation results very well. On the other hand, for one-phase systems the grain size distribution is in excellent agreement with an analytical grain size distribution function based on a statistical mean-field theory of grain growth that is completely compatible with the principal physical condition of total volume conservation.
\end{abstract}

Keywords: Monte Carlo, Grain Growth, Grain Size Distribution, Grain Structure, Polycrystalline Materials

\section{Introduction}

It is well recognized that the microstructure of polycrystalline materials consist of several grains which are typically polyhedral and attached together by interatomic forces. Each grain has a consistent structure and is arranged randomly within solids similar to soap bubbles. The structure formed by the grains is intrinsically unstable. Therefore, the grain structure experiences numerous variations with time which have a strong effect on physical and mechanical properties of the materials. Consequently, understanding how grain structures change and establishing measureable structure property relations are substantial in many theoretical and practical interests.

In the past decades, microstructural evolution of polycrystalline materials has been investigated thoroughly by experiments, theories and computer simulations. The ability to control the properties of polycrystalline materials and enhance their performance depends on the development of analytical material models. Various numerical models have been developed to investigate the microstructural evolution of polycrystalline materials, which have provided a new methodology to bridge the gap between experiments and theories. Examples of these models are classified as followed. Voroni and his modified method [1-3] has been applied to study grain growth and nucleation. Curvature-driven grain growth [4-8] has shown to be successful to simulate growth even though it failed to simulate Ostwald ripening. Continuum thermodynamic techniques [9-11] have been employed fruitfully to study microstructural evolution problems. Cellular automata methods [12] depends on the pixels state near the considered pixel. Monte Carlo Potts model has been developed to study grain growth in one-phase systems [13-21], two-phase solid-liquid systems [22,23], two-phase solid-solid systems [24-26] and three-phase systems [27].

Since the microstructure of polycrystals comprises grains of different sizes, they can be described by an average grain size and a grain size distribution. The average grain size $<R>$ follows the power-growth law given by

$$
<R>^{n}=<R_{0}>^{n}+k t
$$

where, $R_{0}$ is the initial grain size at $t=0, k$ is the grain growth constant, and $n$ is the grain growth exponent, which has a 
theoretical value of $n=2$ for grain growth in one-phase materials [28-30]. In two-phase polycrystalline materials, the value of grain growth exponent is $n=3$ in case of volume diffusion grain growth [31-32] and $n=4$ when grain growth is controlled by grain boundary diffusion [33].

As grain growth continues, the sum of grain sizes keeps the same and the number of grains $N$ decreases according to the following equation,

$$
N(t)=(A t+B)^{-2 / n} .
$$

After an initial transient time period, the grain size distribution, when plotted against the relative grain size $x$ defined as $x=R /<R>$, becomes stationary. Hillert [34] has derived his recognized grain size distribution function which satisfies a continuity equation. However, Hillert's classical grain size distribution function does neither agree with experimental measurements nor with computer simulations. Therefore, much efforts to further develop Hillert's theory were done based on computer simulations taking correlations between nearest neighboring grains into account (e.g., [35-36]). The resulting size distribution functions still satisfy the continuity equation in size space, but are more flexible due to additional parameters.

Zöllner and Streitenberger [37] have derived a grain size distribution function which varies with the relative grain size $\mathrm{x}$ as given by the following expression,

$$
f(x)=a x_{0}^{a} \exp (a) \frac{x}{\left(x_{0}-x\right)^{a+2}} \exp \left(\frac{-a x_{0}}{x_{0}-x}\right) \text {, for } 0 \leq x \leq x_{0}
$$

with the cut-off parameter $\mathrm{x}_{0}$ given by

$$
x_{0}=\frac{a\left(x_{0}\right)^{a\left(x_{0}\right)-1}}{\exp \left(a\left(x_{0}\right)\right) \Gamma\left(a\left(x_{0}\right)-1, a\left(x_{0}\right)\right)}
$$

using the incomplete Gamma-function $\Gamma(a-1, a)$. This analytical grain size distribution, (3), is scaled, normalized, and fulfills the continuity equation. The cut-off parameter $x_{0}$ is the only free adjustable parameter resulting in a very good agreement with 3D Monte Carlo Potts model simulation results [37-38].

In the present work, the Monte Carlo method for two-phase grain growth is employed to study the grain size distribution of two-phase polycrystalline materials. The emphasis of the present work is on the grain size distribution in the self-similar growth regime. The simulated grain size distribution for single-phase systems will be compared with the grain size distribution function derived by Zöllner and Streitenberger [37]; however, the simulated grain size distribution for tow-phase systems will be compared with the normal Gaussian distribution function.

\section{Numerical Implementations}

The Monte Carlo Potts model for two-phase systems [26] has been implemented to simulate the evolution of the grain size distribution for two-phase polycrystalline materials. The structure of two-phase materials is mapped onto a two-dimensional square lattice with a size of $400 \times 400$. Then, the lattices were randomly occupied with the desired volume fractions of both phases.

Every site of phase $\mathrm{A}$ is assigned a random positive number between 1 and $\mathrm{Q}$, and every site of phase $\mathrm{B}$ is assigned a random negative number between -1 and $-Q$. In all our simulations we used the value of $Q=100$. The time unit in these simulations is one Monte Carlo step (MCS) which is proportional to $N$ reorientation attempts where $N$ is the number of lattice sites. The grain boundary energies are identified by significant interaction between nearest neighbor sites. These interactions can be expressed by the following Hamiltonian,

$$
E_{\text {total }}=\frac{1}{2} \sum_{i=1}^{N} \sum_{j=1}^{N} E(i, j)\left[1-\delta\left(q_{i}-q_{j}\right)\right],
$$

where $E(i, j)$ is the boundary energy between site $i$ and $j, q_{i}$ is the spin of the i-th site.

Then the microstructural evolution is simulated by a Monte Carlo approach in which a site is randomly chosen and reoriented to a new different randomly selected configuration between 1 and Q or -1 and -Q. If the energy change $\Delta E$ is less than or equal to zero, then new configuration will be accepted. If $\Delta E$ is positive, a random number $r$ between 0 and 1 is selected and the new configuration will be accepted only if $r \leq$ $\exp \left(-\Delta E / k_{B} T\right)$, where $k_{B}$ is the Boltzman constant and $T$ is the temperature. The temperature has the value of $\mathrm{T}=0.7$, which is high enough to avoid lattice pinning, but also not too high so that the transition to volume diffusion transition regime may not occur. While the value of $\mathrm{T}=0$ is used to simulate curvature grain growth (compare [39-40]).

\section{Simulated Grain Size Distributions}

It was found in a previous work [26] that grain growth in two-phase polycrystalline materials is controlled by grain boundary diffusion and obeys the growth law given in (1) with $n=4$, which is independent of the volume fraction of the second phase. In the present work, we find that the average grain size increases with time and the number of grains drops, where the latter is illustrated by solid lines in Figs. 1 and 2 for a series of different volume fractions. This is a result of grain coarsening, which occurs as a result of vanishing small grains and moving their atoms to the larger neighboring grains. The volume fractions investigated of both phases varies between $0 \% \mathrm{~B}-20 \%$ $\mathrm{B}$ and $80 \% \mathrm{~A}-100 \% \mathrm{~A}$ in Fig. 1 and between 30\% B-50\% B and $50 \%$ A-70\% A in Fig. 2. These simulated curves (solid lines) follow the fit of (2) (dashed lines), where the numeric exponent has a value of -1 in case of the single-phase system $(0 \% \mathrm{~B}$ and $100 \% \mathrm{~A}$ ) and has the value of $-1 / 2$ for two-phase systems. It can be noticed that most grains have disappeared before the structure reaches its self-similar regime; then, eventually approached zero after long aging times. In particular, the self-similar regime is arrived at earlier aging time close to $t \sim$ 1,000 MCS for the one-phase system; however, the self-similar 
regime is arrived at longer aging time between 10,000-100,000 MCS for two-phase systems depending on the volume fraction.

Simultaneously grain growth continues until the self-similar regime is reached, where the grain size distribution is time invariant. The simulated grain size distribution functions $F(R, t)$ are plotted at simulation time $t \approx$ $28,000 \mathrm{MCS}$ as a function of the grain size $R$ in Figs. 3 and 4 for various volume fractions analogously to Figs. 1 and 2 . It can be noticed that the size distributions becomes broader as the volume fraction increases from $10 \%$ to $100 \%$. At small volume fractions of the B-phase, grains are spread at grain boundaries and corners of the A-phase leading to a narrow and peaked distribution. As the volume fraction of the A-phase increases, the grains become interconnected resulting in a wider distribution. This can be observed in Fig. 7 for the simulated microstructures in two-phase systems with different volume fractions. Therefore, these results suggest that there is not a single self-similar size distribution for two-phase systems; but, the size distribution varies with the change of the volume fraction of both phases.

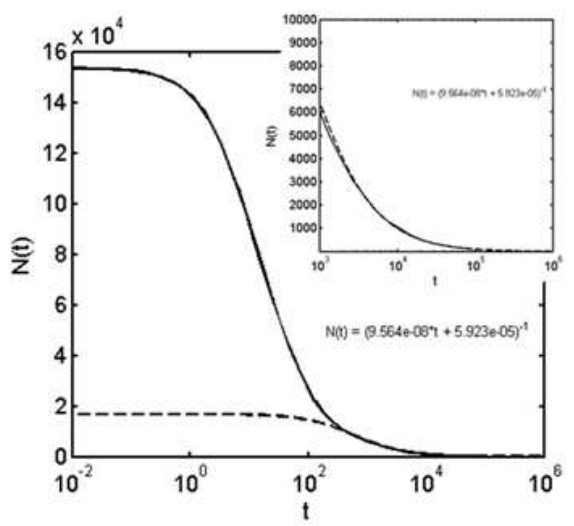

$0 \% \mathrm{~B}, 100 \% \mathrm{~A}$
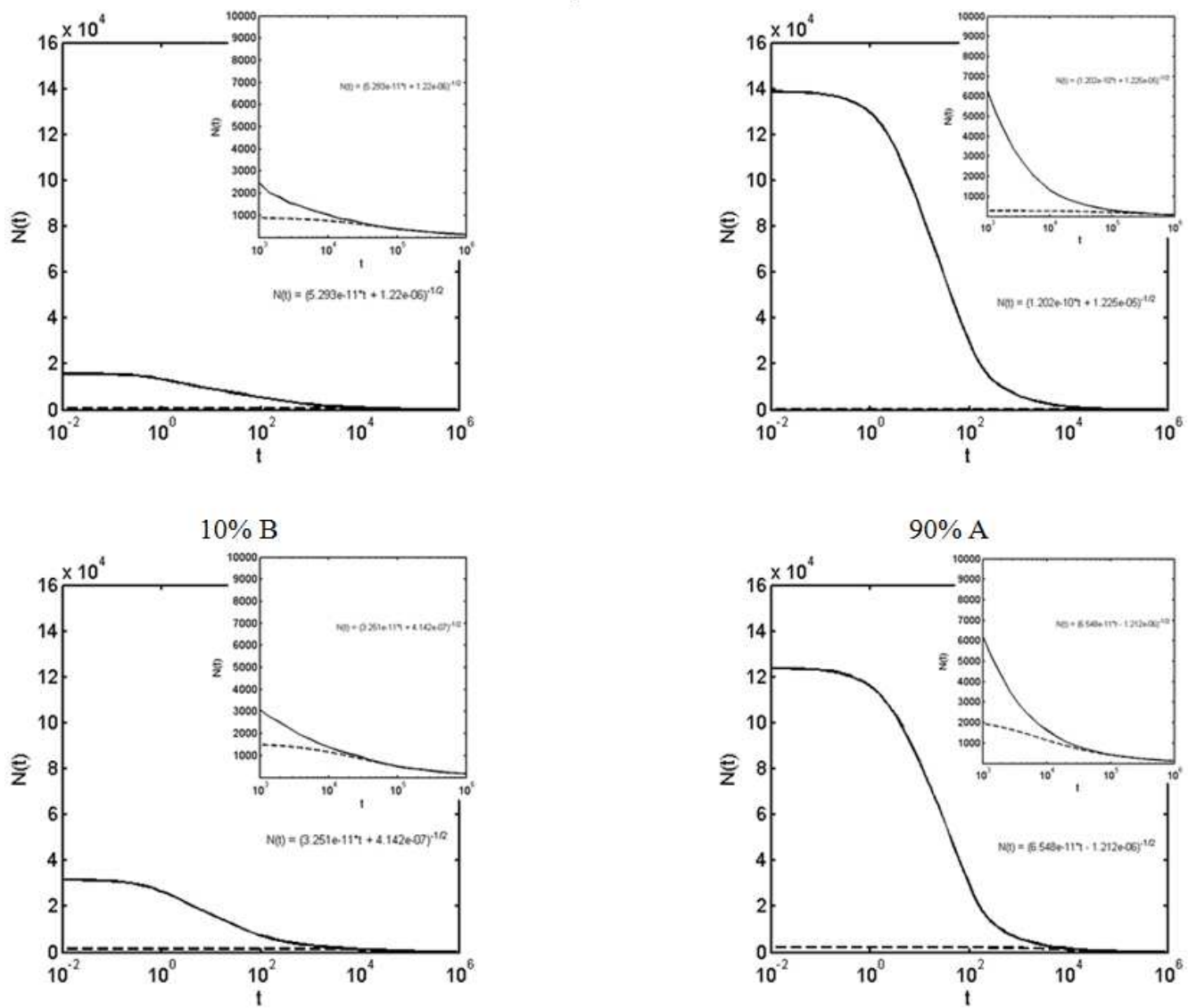

$20 \%$ B

$80 \% \mathrm{~A}$

Figure 1. The number of grains as a function of time (solid line) for various volume fractions, fitting to Equation 2 (dashed line). The volume fractions of both phases varies between $0 \% \mathrm{~B}-20 \% \mathrm{~B}$ and $80 \% \mathrm{~A}-100 \% \mathrm{~A}$ as indicated. 


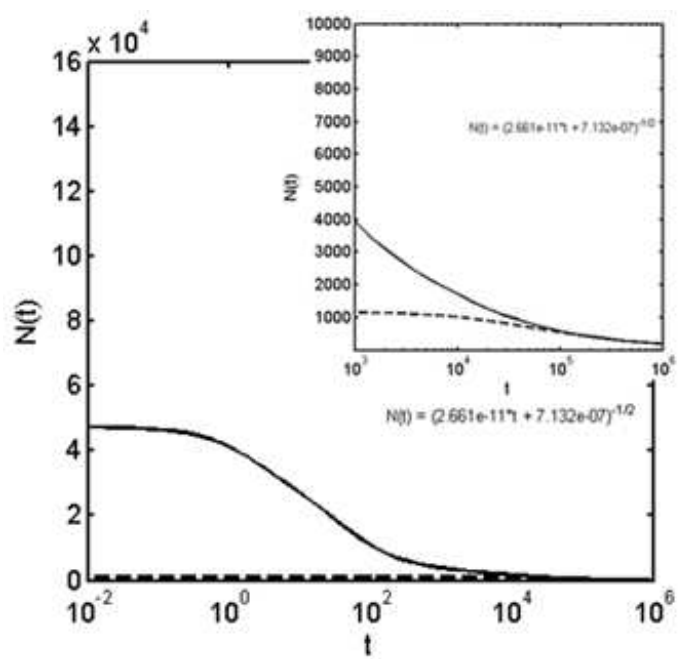

$30 \% \mathrm{~B}$

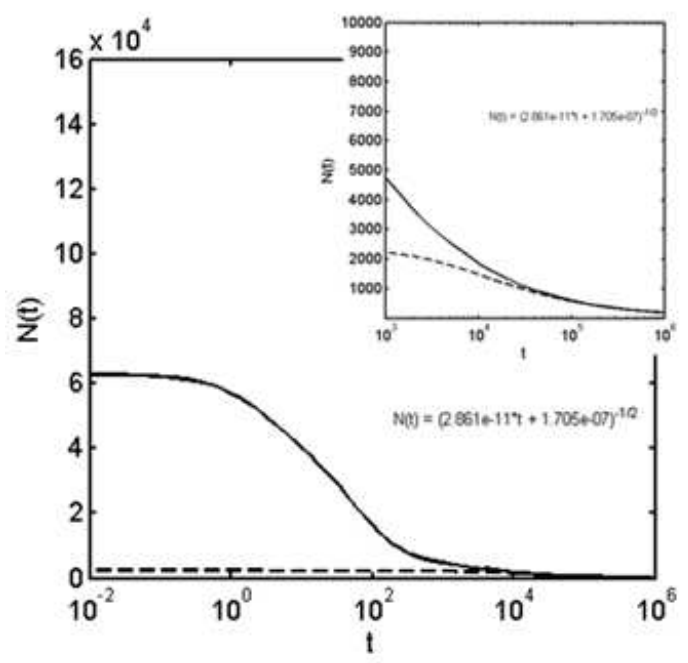

$40 \% \mathrm{~B}$

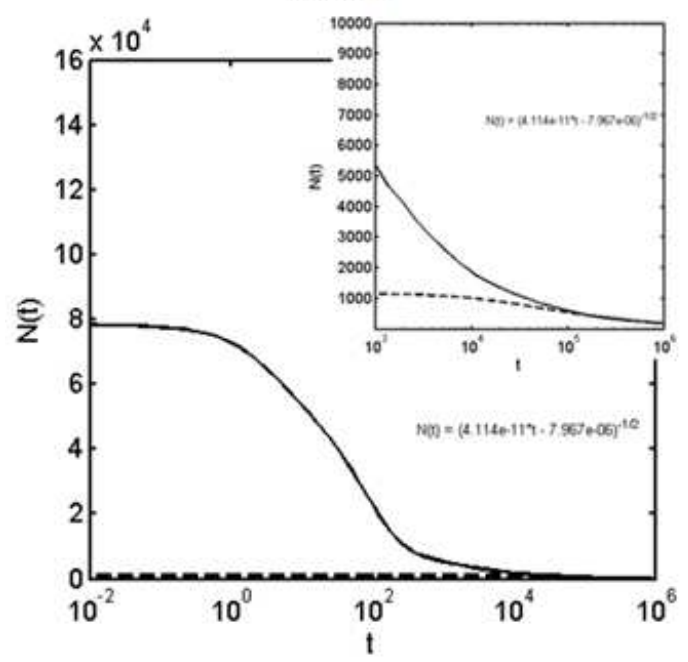

$50 \% \mathrm{~B}$
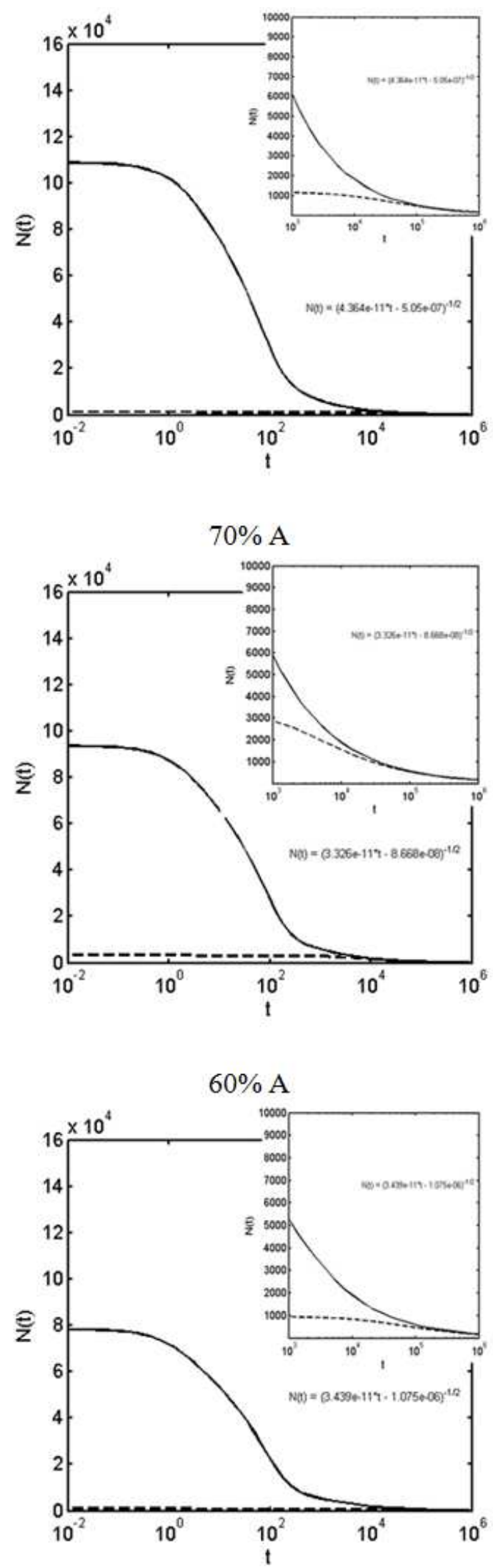

$50 \% \mathrm{~A}$

Figure 2. The number of grains as a function of time (solid line) for various volume fractions, fitting to Equation 2 (dashed line). The volume fractions of both phases varies between $30 \% \mathrm{~B}-50 \% \mathrm{~B}$ and $50 \% \mathrm{~A}-70 \% \mathrm{~A}$ as indicated. 


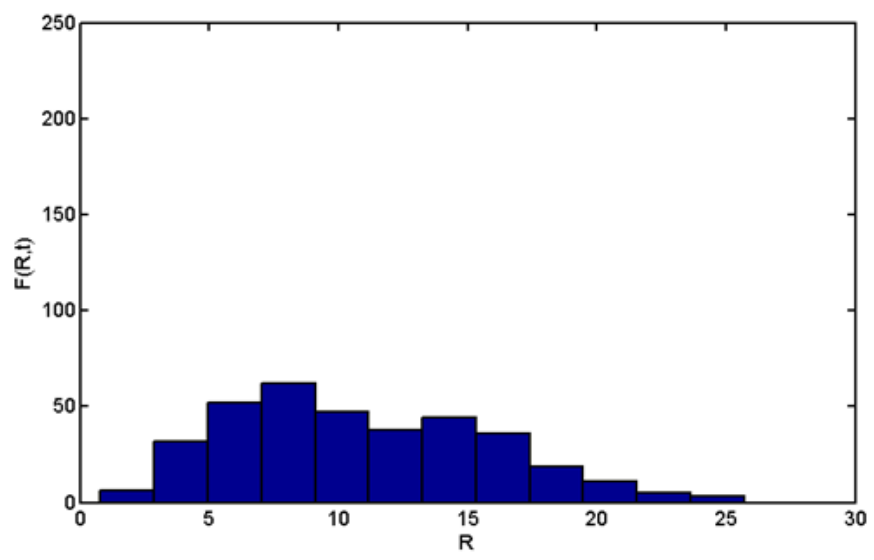

$0 \% \mathrm{~B}, 100 \% \mathrm{~A}$
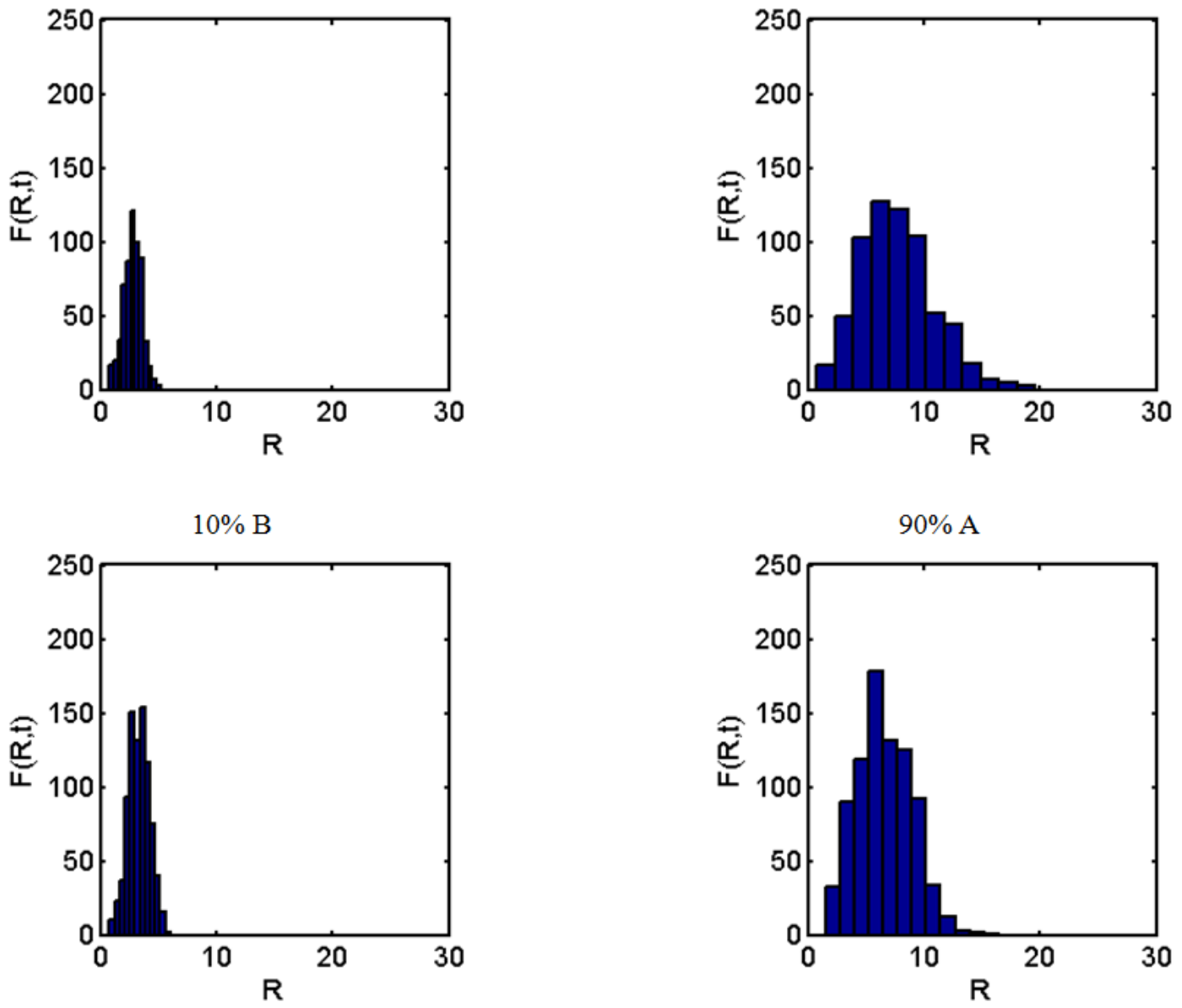

$20 \%$ B

$80 \% \mathrm{~A}$

Figure 3. Grain size distributions in Monte Carlo Potts model simulations of microstructure evolution in two-phase polycrystalline materials performed on a $400 \times 400 \mathrm{grid}$, plotted as a function of the average grain size, $R$, for several volume fractions of both phases at $t \approx 28,000$ MCS. The volume fractions of both phases varies between $0 \% \mathrm{~B}-20 \% \mathrm{~B}$ and $80 \% \mathrm{~A}-100 \% \mathrm{~A}$ as indicated. 

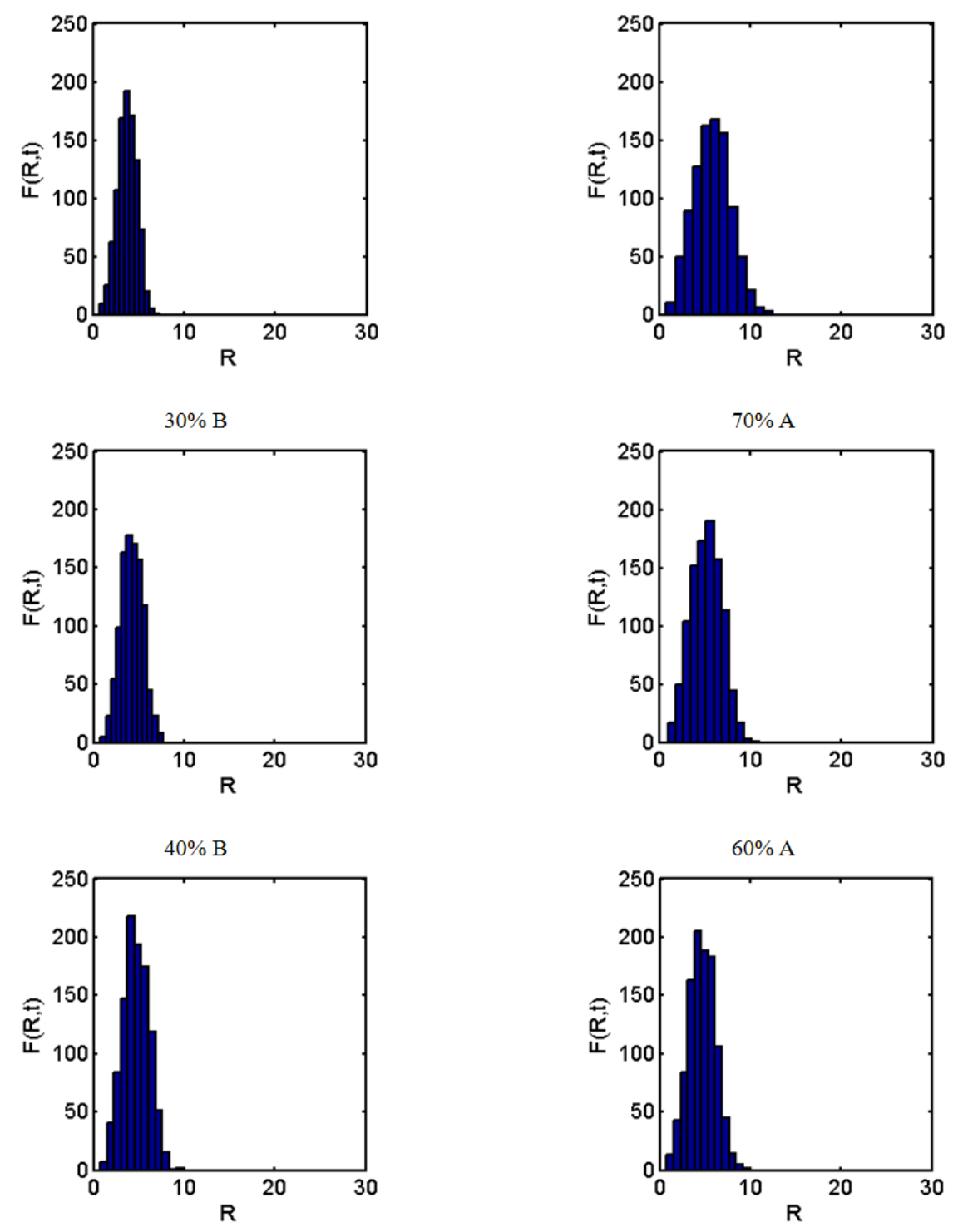

$50 \% \mathrm{~B}$

$50 \% \mathrm{~A}$

Figure 4. Grain size distributions in Monte Carlo Potts model simulations of microstructure evolution in two-phase polycrystalline materials performed on a $400 \times 400 \mathrm{grid}$, plotted as a function of the average grain size, $R$, for several volume fractions of both phases at $t \approx 28,000$ MCS. The volume fractions of both phases varies between $30 \% \mathrm{~B}-50 \% \mathrm{~B}$ and $50 \% \mathrm{~A}-70 \% \mathrm{~A}$ as indicated.

Figs 5 and 6 display the scaled size distribution $f(x)$ versus the normalized grain size $x$ for a series of volume fractions at five different time steps from the self-similar regime. It can be observed that the grain size distribution is indeed self-similar and stationary. The grain size distribution exhibits a peak at $x$ $=1$ and an apparent cut-off between $x=2.0$ and $x=2.5$ depending on the case. In particular, we find that increasing the volume fraction of phase B from $10 \%$ to $50 \%$ changes the scaled distribution of phase $\mathrm{B}$ from narrow and peaked to a broader distribution resulting for $50 \%$ B-phase in a distribution that is more or less identical to the distribution for $50 \%$ A-phase. Furthermore, the simulated grain size distribution in the self-similar regime can be well described by a fit of the theoretical grain size distribution given by (3) for the one-phase system. However, we find that the grain size distribution within the self-similar regime for two-phase systems can be described very well by the normal Gaussian distribution. 


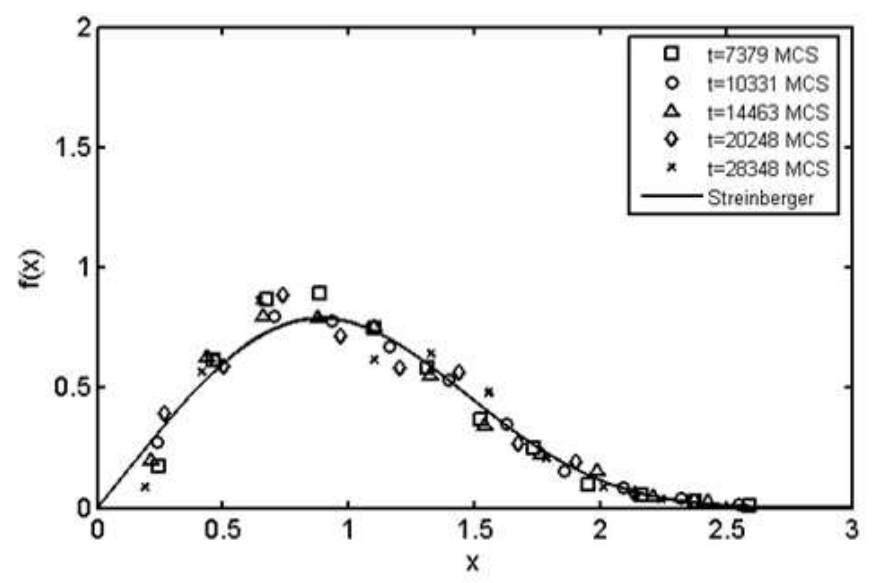

$0 \% \mathrm{~B}, 100 \% \mathrm{~A}$
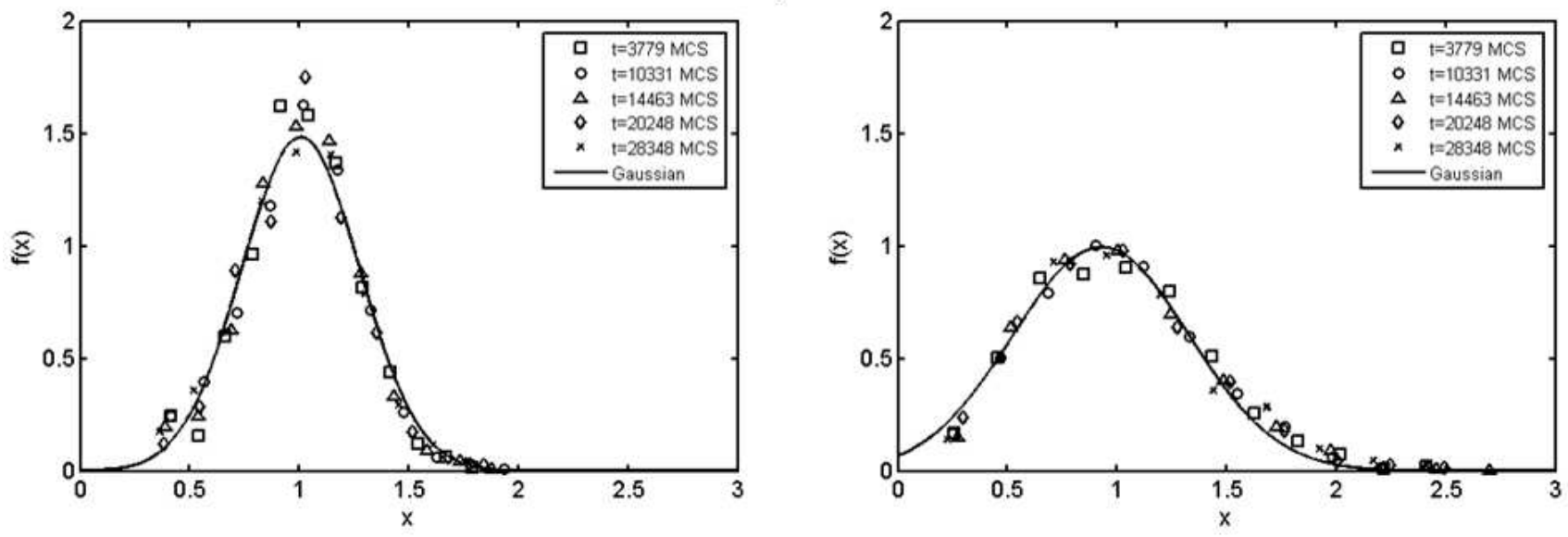

$10 \% \mathrm{~B}$

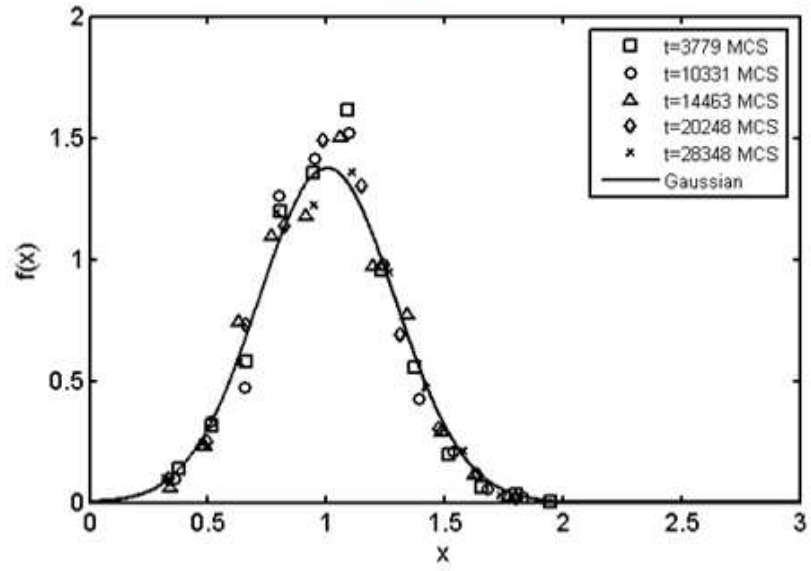

$20 \%$ B

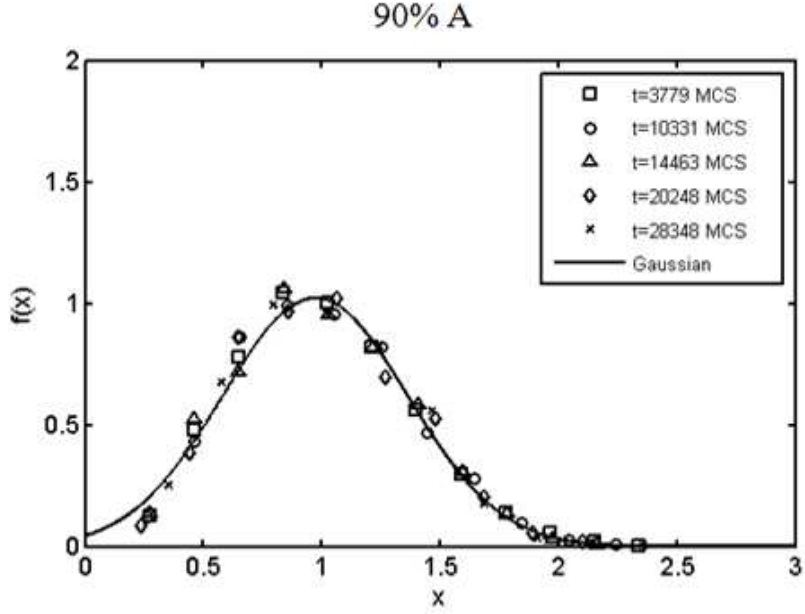

$80 \%$ A

Figure 5. Time dependence of grain size distribution on the volume fraction for five different time steps, comparing with the theoretical expression Equation (3) for one-phase system and with the normal Gaussian distribution function for two-phase system. The volume fractions of both phases varies between $0 \%$ B-20\% $B$ and $80 \% \mathrm{~A}-100 \% \mathrm{~A}$ as indicated. 

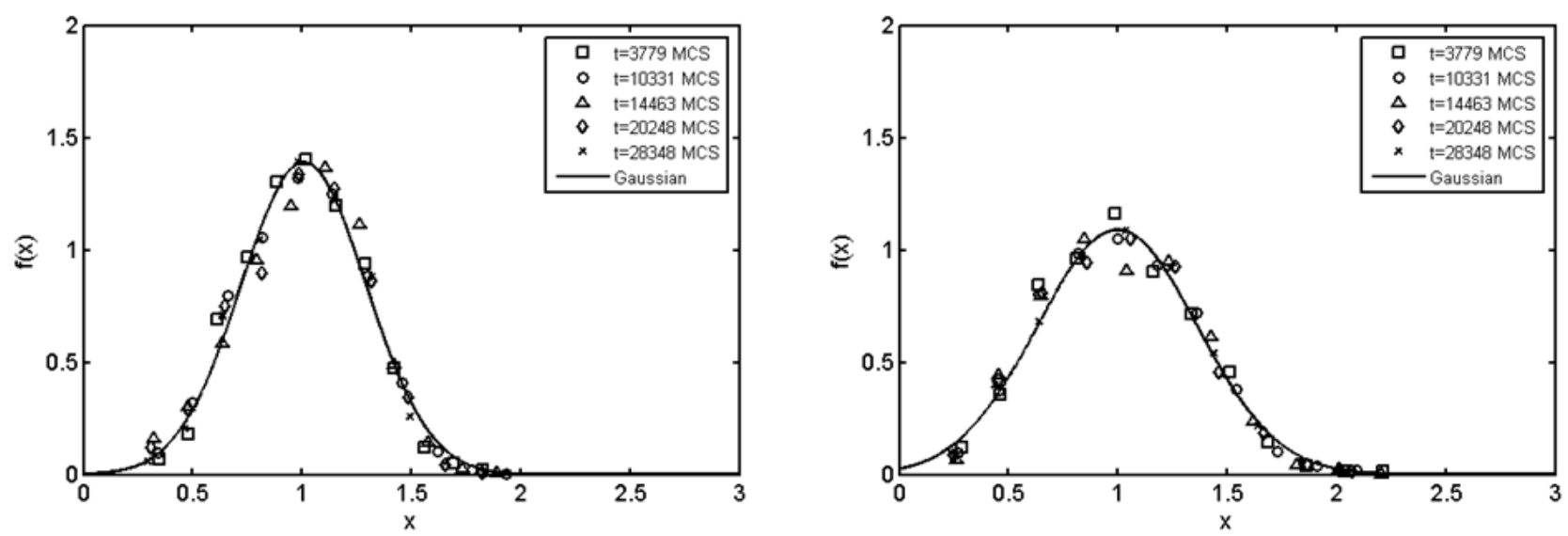

$30 \% \mathrm{~B}$
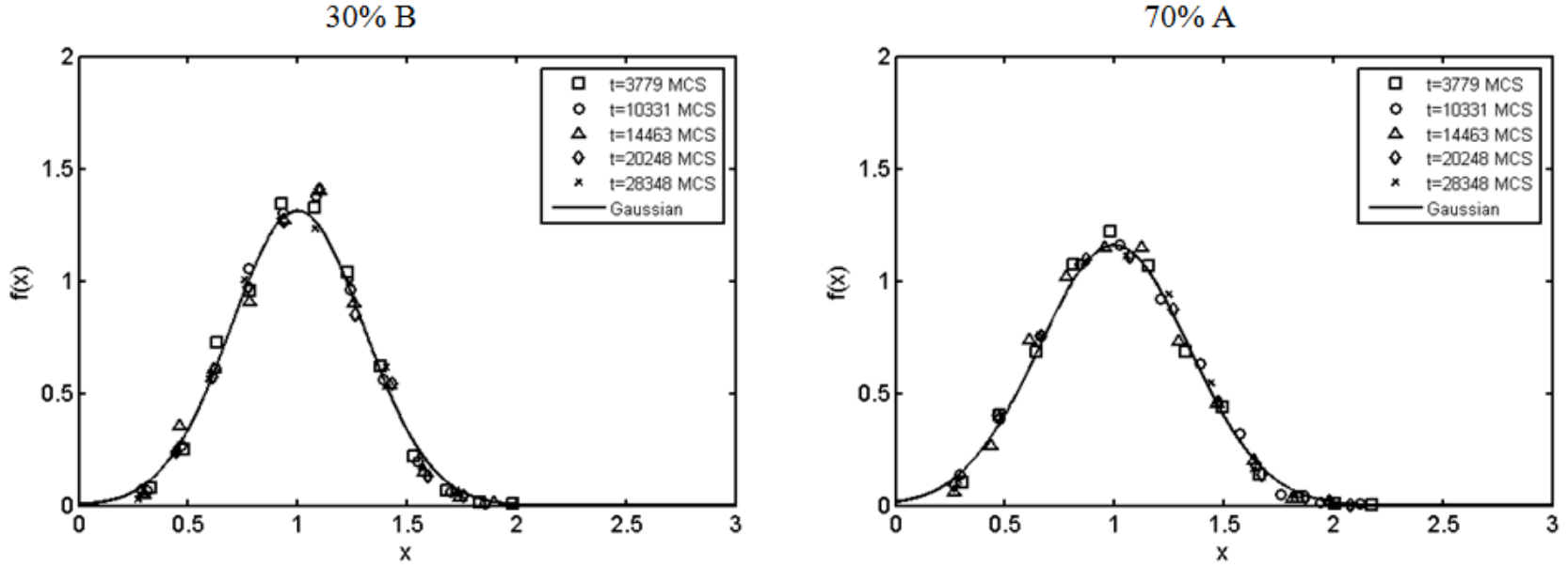

$40 \% \mathrm{~B}$



$50 \% \mathrm{~B}$

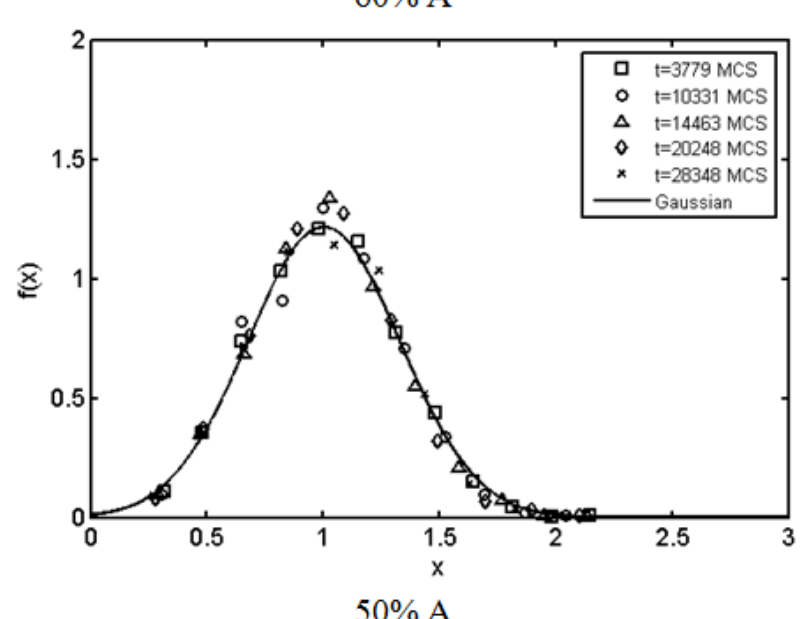

Figure 6. Time dependence of grain size distribution on the volume fraction for five different time steps, comparing with the theoretical expression Equation (3) for one-phase system and with the normal Gaussian distribution function for two-phase system. The volume fractions of both phases varies between $30 \%$ B-50\% $B$ and $50 \% A-70 \% A$ as indicated. 


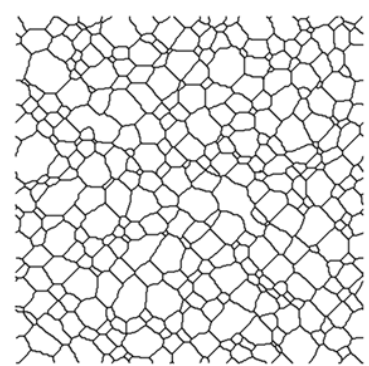

$0 \% \mathrm{~B}, 100 \mathrm{~A}$

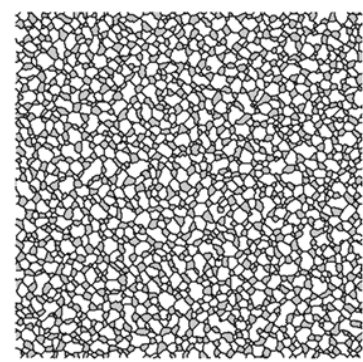

$30 \% \mathrm{~B}, 70 \mathrm{~A}$

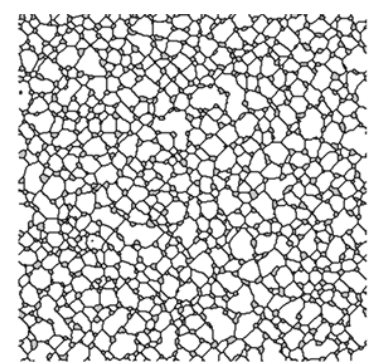

$10 \% \mathrm{~B}, 90 \mathrm{~A}$



$40 \% \mathrm{~B}, 60 \mathrm{~A}$

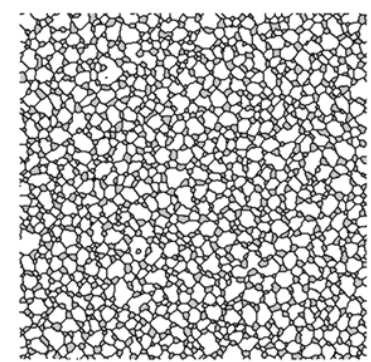

$20 \% \mathrm{~B}, 80 \mathrm{~A}$

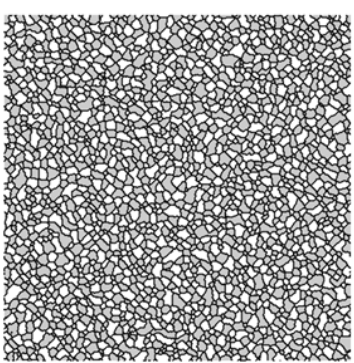

$50 \% \mathrm{~B}, 50 \mathrm{~A}$

Figure 7. Microstructural evolution in two-phase system at $t \approx 28,000$ MCS for different volume fractions as specified. Grains of phase A are white and grains of phase $B$ are gray.

\section{Conclusion}

The grain size distribution in two-phase polycrystalline materials is simulated for a broad series of second phase volume fractions through computer simulations based on the Monte Carlo Potts model. It is found that the grain size distribution changes significantly with the volume fraction. The grain size distribution becomes wider and lower peaked with increasing volume fraction of the second phase, where the normal Gaussian distribution function is in a good agreement with the simulation results of the grain size distribution for all two-phase systems. However, for the one-phase system the grain size distribution is in excellent agreement with a non-Gaussian, analytical grain size distribution function derived in a previous work [37] and expressed by Equation (3). Since there is no theoretical model for the grain size distribution functions in two-phase system yet, an analytical distribution function needs to be developed to describe two-phase grain size systems in the future.

\section{References}

[1] K.W. Mahis, K. Hanson and J.W. Morrid Jr., "Comparative analysis of the cellular and Johnson-Mahl microstructures through computer simulation," Act Metall., Vol. 28, pp. 443-453, 1980.

[2] H.J. Frost and C.V. Thompson, "The effect of nucleation conditions on the Topology and Geometry of two-dimensional grain structures," Act Metall., vol. 35, pp. 529-540, 1987.

[3] O. Ito and E.R. Fuller Jr., "Computer modeling of anisotropic grain microstructure in two-dimensions," Act Metall., vol. 41, pp. 191-198, 1993.

[4] E. Schule, "A justification of the Hillert distributions by spatial grain growth simulation performed by modifications of Laguerre tessellations," Comput. Mater. Sci., vol. 5, pp. 277-285, 1996.

[5] H.J. Frost, C.V. Thompson, C.L. Howe, and J. Whang, "A two-dimensional computer simulation of capillarity-driven grain growth: Preliminary results," Scr. Metal., vol. 22, pp. $65-70,1988$.

[6] E.A. Ceppi and B.O.Nasello, "Computer simulation of bidimensional grain growth," Scr. Metal., vol. 12, pp. 1221-1225, 1984.

[7] D. Kinderlehrer, J. Lee, I. Livshits, A. Rollett and S. Ta'asan, "Mesoscale simulation of grain growth," Materials Science Forum, Vol. 467, pp. 1057-1062, 2004.

[8] L. A. Barrales-Mora, V. Mohles, P. J. Konijnenberg and D. A. Molodov, "A novel implementation for the simulation of 2-D grain growth with consideration to external energetic fields," Comput. Mater. Sci., Vol. 39, pp. 160-165, 2007.

[9] D. Fan and L.Q. Chen, "On the possibility of spinodal decomposition in zirconia-yittria alloys-A theoretical investigation," J. Am. Ceram., vol. 78, pp. 1680-1686, 1995.

[10] Z. Nikolic and W. Huppmann, "Computer simulation of chemically driven grain growth during liquid phase sintering," Acta Metall., vol. 28, pp. 475-479, 1980.

[11] P.W. Voorhees and M.E. Glicksman, "Solution to the multi-particle diffusion problem with applications to Ostwald-ripening-II. Computer simulation," Acta Metall., vol. 32, pp. 2013-2030, 1984.

[12] J. Geiger, A. Roosz and P. Barkoczy, "Simulation of grain coarsening in two dimensions by cellular-automaton," Acta Mater., Vol. 49, pp. 623-629, 2011.

[13] M.P. Anderson, D.J. Srolovitz, G.S. Grest, and P.S. Sahni, "Computer simulation of grain gowth-I. Kinetics," Acta Metall. Vol. 32, pp. 783-791, 1984. 
[14] M.P. Anderson, G.S. Grest, D.J. Srolovitz, "Computer simulation of grain gowth in three dimensions," Phil. Mag. Vol. 59B, pp. 293-329, 1989.

[15] X. Song and G. Liu, "A simple and efficient three-dimensional Monte Carlo simulation of grain growth," Scr. Mater., Vol. 38, pp. 1691-1696, 1998.

[16] P. Blikstein and A. P. Tschiptschin, "Monte Carlo simulation of grain growth, " Materials Research, Vol. 2, 133-137, 1999.

[17] Q. Yu and S.K. Esche, "A Monte Carlo algorithm for single phase normal grain growth with improved accuracy and efficiency," Comput. Mater. Sci., Vol. 27, pp. 259-270, 2003.

[18] L. Hui, W. Guanghou, D. Feng, B. Xiufang, and F. Pederiva, "Monte Carlo simulation of three-dimensional polycrystalline material," Materials Science and Engineering: A, Vol. 357, pp. $153-158,2003$.

[19] Q. Yu and S.K. Esche, "Three-dimensional grain growth modeling with a Monte Carlo algorithm," Materials Letters, Vol. 57, pp. 4622-4626, 2003.

[20] C. Ming Huang, C.L. Joanne, B.S.V. Patnaik, and R. Jayaganthan, "Monte Carlo simulation of grain growth in polycrystalline materials," Applied Surface Science, Vol. 252, pp. 3997-4002, 2006.

[21] D.Zöllner and P. Streitenberger, "Monte Carlo Simulation of Normal Grain Growth in Three Dimensions," Materials Science Forum, Vol. 567, pp. 81-84, 2008.

[22] V. Tikare and J.D. Gawlez, "Applications of the Potts model to simulation of Ostwald ripening," J. Am. Ceramic Soc., vol. 81, pp. 485-491, 1998.

[23] R. M. German, P. Suri, and S.J. Park, "Review: liquid phase sintering," Journal of materials science, Vol. 44, pp. 1-39, 2009.

[24] R. El-Khozondar, H. El-Khozondar, G. Gottstein, A. Rollet, "Microstructural Simulation of Grain Growth in Two-phase Polycrystalline Materials," Egypt. J. Solids, vol. 29(1), pp. 35-47, 2006.

[25] R. El-Khozondar and H. El-Khozondar, "Numerical simulations of coarsening of lamellar structures: applications to metallic alloys," Egypt. J. Solids, vol. 27(2), pp.189-199, 2004.

[26] V.S. Solomatov, R. El-Khozondar, and V. Tikare, "Grain size in the lower mantle: constraints from numerical modeling of grain growth in two-phase systems," Phys. Earth. Planet. Inter., vol. 129, pp. 265-282, 2002.
[27] R. El-Khozondar and H. El-Khozondar, "Numerical modeling of microstructural evolution in three-phase polycrystalline materials," Arabian Journal for Science and Engineering, vol. 34(1A), pp. 241-252, 2009.

[28] J.E. Burke, D. Turnbull, "Recrystallization and grain growth," Prog. Metal. Phys., vol. 3, pp. 220-292, 1952.

[29] P. Felthman, "Grain growth in metals," Acta Metall., vol. 5, pp. 97-105, 1957.

[30] N.P. Louat, "On the theory of grain growth," Acta Metall., vol. 22, pp. 721-724, 1974.

[31] M. Hillert, "On the theory of normal and abnormal grain growth," Acta Metall., vol. 13, pp. 227-238, 1965.

[32] I.M. Lifshitz and V.V. Slyozov, "The kinetics of precipitation from supersaturated solid solution," J. Phys. Chem. Solids, vol. 19, pp. 35-50, 1961.

[33] C. Wagner, "Theorio der altering von niederschlägen durch unlösen," Elektrochem., vol. 65, pp. 581-591, 1961.

[34] A.J. Ardell, "On the coarsening of grain boundary," Acta Metall., vol. 20, pp. 601-609, 1972.

[35] P. Streitenberger, "Generalized Lifshitz-Slyozov theory of grain and particle coarsening for arbitrary cut-off parameter," Scr. Mater., vol. 39, pp. 1719-1724, 1998.

[36] M.W. Nordbakke, N. Ryum, and O. Hunderi, "Curvilinear polygons, finite circle packings, and normal grain growth," Materials Science and Engineering A., vol. 385, pp. 229-234, 2004.

[37] D. Zöllner and P. Streitenberger, "Three-dimensional normal grain growth: Monte Carlo Potts model simulation and analytical mean field theory," Scr. Mater., vol. 54, pp. 1697-1702, 2006.

[38] D. Zöllner and P. Streitenberger, "Grain size distributions in normal grain growth," Practical Metallography/Praktische Metallographie," vol. 47, pp. 618-639, 2010.

[39] V. Tikare and J. D. Cawley, "Numerical simulation of grain growth in liquid phase sintered materials. II. Study if isotropic grain growth," Acta Mater., vol. 46, pp. 1343-1356, 1998.

[40] D. Zöllner, "A new point of view to determine the simulation temperature for the Potts model simulation of grain growth," Comput. Mater. Sci., vol. 86, pp. 99-107, 2014. 